\title{
COMPUTATIONAL EXPERIMENTS WITH ABS ALGORITHMS FOR KKT LINEAR SYSTEMS 円
}

\author{
E. Bodon [4, A. Del Popolo [3, L. Lukšan f and E. Spedicato 目
}

\section{Introduction}

In this report, we present numerical experiments with three particular ABS algorithms:

(1) The Huang or modified Huang algorithm,

(2) The implicit LU algorithm,

These algorithms have been used for finding a solution of the linear KKT system

$$
\left[\begin{array}{cc}
B & A^{T} \\
A & 0
\end{array}\right]\left[\begin{array}{l}
x \\
y
\end{array}\right]=\left[\begin{array}{l}
b \\
c
\end{array}\right],
$$

where $A \in R^{m, n}, B \in R^{n, n}$ is symmetric, $b \in R^{n}, c \in R^{m}, x \in R^{n}, y \in R^{m}$ and $m \leq n$.

The considered algorithms belong to the basic ABS class of algorithms for solving a linear system $A x=b$, which is a special case of the scaled ABS class, given by the following scheme:

(A) Let $x_{1} \in R^{n}$ be arbitrary and $H_{1} \in R^{n, n}$ be nonsingular arbitrary. Set $i=1$.

(B) Compute the residual $r_{i}=A x_{i}-b$. If $r_{i}=0$, then stop, $x_{i}$ solves the problem. Otherwise compute $s_{i}=H_{i} A^{T} v_{i}$, where $v_{i} \in R^{n}$ is arbitrary, save that $v_{1}, \ldots, v_{i}$ are linearly independent. If $s_{i} \neq 0$, then go to (C). If $s_{i}=0$ and $r_{i}^{T} v_{i}=0$, then set $x_{i+1}=x_{i}, H_{i+1}=H_{i}$ and go to $(\mathrm{F})$. If $s_{i}=0$ and $r_{i}^{T} v_{i} \neq 0$, then stop, the system is incompatible.

(C) Compute the search vector $p_{i}$ by

$$
p_{i}=H_{i}^{T} z_{i}
$$

where $z_{i} \in R^{n}$ is arbitrary, save that $z_{i}^{T} H_{i} A^{T} v_{i} \neq 0$.

(D) Update the estimate of the solution by

$$
x_{i+1}=x_{i}-\alpha_{i} p_{i},
$$

where the stepsize $\alpha_{i}$ is given by

$$
\alpha_{i}=r_{i}^{T} v_{i} / p_{i}^{T} A^{T} v_{i}
$$

\footnotetext{
${ }^{1}$ Work partly supported by grant GA CR 201/00/0080 and by MURST 1997 and 1999 Programmi di Cofinanziamento

${ }^{2}$ Department of Mathematics, University of Bergamo, Bergamo 24129, Italy (bodon@unibg.it)

${ }^{3}$ Department of Mathematics, University of Bergamo, Bergamo 24129, Italy (delpopolo@unibg.it)

${ }^{4}$ Institute of Computer Science, Academy of Sciences of the Czech Republic, Pod vodárenskou věží 2, 18207 Prague 8, Czech Republic (luksan@uivt.cas.cz)

${ }^{5}$ Department of Mathematics, University of Bergamo, Bergamo 24129, Italy (emilio@unibg.it)
} 
(E) Update the Abaffian matrix by

$$
H_{i+1}=H_{i}-H_{i} A^{T} v_{i} w_{i}^{T} H_{i} / w_{i}^{T} H_{i} A^{T} v_{i},
$$

where $w_{i} \in R^{n}$ is arbitrary, save that $w_{i}^{T} H_{i} A^{T} v_{i} \neq 0$.

(F) If $i=m$, then stop, $x_{i+1}$ solves the problem. Otherwise increment the index $i$ by one and go to $(\mathrm{B})$.

From (E), it follows by induction that $H_{i+1} A^{T} V_{i}=0$ and $H_{i+1}^{T} W_{i}=0$, where $V_{i}=\left[v_{1}, \ldots, v_{i}\right]$ and $W_{i}=\left[w_{1}, \ldots, w_{i}\right]$. One can show that the implicit factorization $V_{i}^{T} A P_{i}=L_{i}$ holds, where $P_{i}=\left[p_{1}, \ldots, p_{i}\right]$ and $L_{i}$ is nonsingular lower triangular. Moreover the general solution of the scaled subsystem $V_{i}^{T} A x=V_{i}^{T} b$ can be expressed in the form

$$
x=x_{i}+H_{i}^{T} q
$$

where $q \in R^{n}$ is arbitrary (see [2] for the proof).

The basic ABS class is the subclass of the scaled ABS class obtained by taking $v_{i}=e_{i}, e_{i}$ being the $i$-th unitary vector ( $i$-th column of the unit matrix). In this case, residual $r_{i}=A x_{i}-b$ need not be computed in (B), $i$-th element $r_{i}^{T} e_{i}=a_{i}^{T} x_{i}-b_{i}$ suffices.

This report is organized as follows. In Section 2, a short description of individual algorithms is given. Section 3 contains some details concerning test matrices and numerical experiments. Section 4 discusses the results of the numerical experiments. The Appendix contains all numerical results. For a listing of the ABS source codes see [12]. For other numerical results on ABS methods see $[1,11]$.

\section{ABS algorithms for KKT equations}

In this report, we use three linear ABS algorithms, two belonging to the basic ABS class and one belonging to the scaled ABS class, for solving the KKT linear systems exploiting their structure. To simplify description, we will assume that $A \in R^{m, n}, m \leq n$, has full row rank so that $s_{i} \neq 0$ in (B). First we describe the used linear solvers.

The Huang algorithm is obtained by the parameter choices $H_{1}=I, v_{i}=e_{i}, z_{i}=a_{i}, w_{i}=a_{i}$. Therefore

$$
p_{i}=H_{i} a_{i}
$$

and

$$
H_{i+1}=H_{i}-p_{i} p_{i}^{T} / a_{i}^{T} p_{i},
$$

From (2) and (3), it follows by induction that $p_{i} \in \operatorname{Range}\left(A_{i}\right)$ and

$$
H_{i+1}=I-P_{i} D_{i}^{-1} P_{i}^{T} .
$$

where $A_{i}=\left[a_{1}, \ldots, a_{i}\right], P_{i}=\left[p_{1}, \ldots, p_{i}\right]$ and $D_{i}=\operatorname{diag}\left(a_{1}^{T} p_{1}, \ldots, a_{i}^{T} p_{i}\right)$. Moreover $H_{i}$ is symmetric, positive semidefinite and idempotent (it is the orthogonal projection matrix into $\left.\operatorname{Null}\left(A_{i-1}\right)\right)$. Since the requirement $p_{i} \in \operatorname{Null}\left(A_{i-1}\right)$ is crucial, we can improve orthogonality by iterative refinement $p_{i}=H_{i}^{j} a_{i}, j>1$ (usually $j=2$ ), obtaining the modified Huang algorithm.

The Huang algorithm can be used for finding the minimum-norm solution to the compatible underdetermined system $A x=b$, i.e. for minimizing $\|x\|$ s.t. $A x=b$. To see this, we use the 
Lagrangian function and convexity of $\|x\|$. Then $x$ is a required solution if and only if $x=A^{T} u$ for some $u \in R^{m}$ or $x \in \operatorname{Range}\left(A^{T}\right)$. But

$$
x_{m+1}=x_{1}-\sum_{i=1}^{m} \alpha_{i} p_{i}
$$

by (D) and $p_{i} \in \operatorname{Range}\left(A_{i}\right) \subset \operatorname{Range}\left(A^{T}\right)$, so that if $x_{1}=0$, then $x_{m+1} \in \operatorname{Range}\left(A^{T}\right)$.

A short description of two versions of the Huang and modified Huang algorithms follows.

\section{Algorithm 1}

(Huang and modified Huang, formula (3).

Set $x_{1}=0$ and $H_{1}=I$.

For $i=1$ to $m$ do

Set $p_{i}=H_{i} a_{i}$ (Huang) or

$p_{i}=H_{i}\left(H_{i} a_{i}\right)$ (modified Huang),

$d_{i}=a_{i}^{T} p_{i}$ and $x_{i+1}=x_{i}-\left(\left(a_{i}^{T} x_{i}-b_{i}\right) / d_{i}\right) p_{i}$.

If $i<m$, then set $H_{i+1}=H_{i}-p_{i} p_{i}^{T} / d_{i}$.

end do

\section{Algorithm 2}

(Huang and modified Huang, formula (4).

Set $x_{1}=0$ and $P_{0}$ empty.

For $i=1$ to $m$ do

Set $p_{i}=\left(I-P_{i-1} D_{i-1}^{-1} P_{i-1}^{T}\right) a_{i}$ (Huang) or

$p_{i}=\left(I-P_{i-1} D_{i-1}^{-1} P_{i-1}^{T}\right)\left(I-P_{i-1} D_{i-1}^{-1} P_{i-1}^{T}\right) a_{i}$ (modified Huang),

$d_{i}=a_{i}^{T} p_{i}$ and $x_{i+1}=x_{i}-\left(\left(a_{i}^{T} x_{i}-b_{i}\right) / d_{i}\right) p_{i}$.

If $i<m$, then set $P_{i}=\left[P_{i-1}, p_{i}\right]$.

end do

The implicit LU algorithm is obtained by the parameter choices $H_{1}=I, v_{i}=e_{i}, z_{i}=e_{i}$, $w_{i}=e_{i}$. Since $W_{i}^{T} H_{i+1}=\left[I_{i}, 0\right]^{T} H_{i+1}=0$, the first $i$ rows of the Abaffian matrix $H_{i+1}$ must be zero. More precisely, the Abaffian matrix has the following structure, with $K_{i} \in R^{n-i, i}$

$$
H_{i+1}=\left[\begin{array}{cc}
0 & 0 \\
K_{i} & I_{n-i}
\end{array}\right]
$$

Only the matrix $K_{i}$ has to be updated. Expression (5) implies that only the first $i$ components of the vector $p_{i}=H_{i}^{T} e_{i}$ can be nonzero and the $i$-th component is unity. Hence the matrix $P_{i}$ is unit upper triangular so that the implicit factorization $A=L P^{-1}$ is of the LU type with units on the diagonal.

A short description of two versions of the implicit LU algorithm follows (the second version is also called the implicit LX algorithm).

\section{Algorithm 3}

(Implicit LU with explicit column interchanges).

Set $x_{1}=0$ and $H_{1}=I$.

For $i=1$ to $m$ do

Set $s_{i}=H_{i} a_{i}$

(only $(i-1)(n-i+1)$ nonzero elements of $H_{i}$ are used). 
Determine $d_{i}=\left|s_{k_{i}}\right|=\max \left(\left|s_{i}^{T} e_{j}\right|, j=1, \ldots, n\right)$.

(only $n-i+1$ nonzero elements of $s_{i}$ are used).

If $k_{i} \neq i$, then swap columns of $A$ and elements of $x$ and $s$ with these indices.

Set $x_{i+1}=x_{i}-\left(\left(a_{i}^{T} x_{i}-b_{i}\right) / d_{i}\right) H_{i}^{T} e_{i}$

(only $i$ nonzero elements of $x_{i}$ are updated).

If $i<m$, then set $H_{i+1}=H_{i}-s_{i} e_{i}^{T} H_{i}^{T} / d_{i}$

(only $i(n-i)$ nonzero elements of $H_{i+1}$ are updated).

end do

\section{Algorithm 4}

(Implicit LX algorithm, or implicit LU with implicit column interchanges).

Set $x_{1}=0, H_{1}=I$.

For $i=1$ to $m$ do

Set $s_{i}=H_{i} a_{i}$

(only $(i-1)(n-i+1)$ nonzero elements of $H_{i}$ are used).

Determine $d_{k_{i}}=\left|s_{k_{i}}\right|=\max \left(\left|s_{i}^{T} e_{j}\right|, j=1, \ldots, n\right)$.

(only $n-i+1$ nonzero elements of $s_{i}$ are used).

Set $x_{i+1}=x_{i}-\left(\left(a_{i}^{T} x_{i}-b_{i}\right) / d_{k_{i}}\right) H_{i}^{T} e_{k_{i}}$

(only $i$ nonzero elements of $x_{i}$ are updated).

If $i<m$, then set $H_{i+1}=H_{i}-s_{i} e_{k_{i}}^{T} H_{i}^{T} / d_{k_{i}}$

(only $i(n-i)$ nonzero elements of $H_{i+1}$ are updated).

end do

We now consider how to use the above algorithm for solving KKT systems, exploiting their structure. Consider the linear KKT system

$$
\begin{gathered}
B x+A^{T} y=b, \\
A x=c .
\end{gathered}
$$

The underdetermined equation (7) can be solved by an arbitrary ABS method obtaining the particular solution $x_{m+1}$. Since $H_{m+1} A^{T}=0$, we get

$$
H_{m+1} B x=H_{m+1} b,
$$

by multiplying (6) by $H_{m+1}$. The coupled system (7)-(8) is overdetermined but compatible so that its unique solution can be found by an arbitrary ABS method. Since $\operatorname{rank}\left(H_{m+1}\right)=n-m$, $m$ equations will be eliminated. Notice that solution of (7)-(8) is obtained in two steps. First we solve (7) to obtain $x_{m+1}$ and $H_{m+1}$. Then we construct $H_{m+1} B$ and $H_{m+1} b$ and starting with $x_{m+1}$ and $H_{m+1}$ we continue with the ABS method to solve (8).

If we use the implicit LU algorithm, then matrix $H_{m+1}$ has the special form (3) and the first $m$ equations of (8) are trivially satisfied, leaving a determined system consisting of (7) and the equation

$$
S_{m} B x=S_{m} b,
$$

where $S_{m}=\left[K_{m}, I_{n-m}\right]$. Moreover, substituting the general solution, see (1)

$$
x=x_{m+1}+H_{m+1}^{T} q,
$$

with $q \in R^{n}$ arbitrary into (6) and using the special form of $H_{m+1}$, we obtain

$$
\left[\begin{array}{cc}
0 & 0 \\
0 & S_{m} B S_{m}^{T}
\end{array}\right]\left[\begin{array}{l}
q_{1} \\
q_{2}
\end{array}\right]=\left[\begin{array}{c}
0 \\
S_{m}\left(b-B x_{m+1}\right)
\end{array}\right] .
$$


Thus $q_{1}$ can be chosen arbitrarily, e.g. $q_{1}=0$, and $q_{2}$ is a solution to the $n-m$ dimensional system

$$
S_{m} B S_{m}^{T} q_{2}=S_{m}\left(b-B x_{m+1}\right),
$$

which can be solved by any ABS method.

Once $x$ is determined, we obtain $y$ by solving the compatible overdetermined system $A^{T} y=$ $b-B x$. Since the equation $A x=c$ was solved beforehand, we are in the same position as in the case of the least-squares solution of an overdetermined linear system. Therefore, using $P_{m}$, we can construct the lower triangular matrix $L_{m}=A P_{m}$ and get the solution from the equation $L_{m}^{T} y=P_{m}^{T}(b-B x)$.

\section{Description of computational experiments}

Performance of ABS algorithms has been tested by using several types of ill-conditioned matrices. These matrices can be classified in the following way. The first letter distinguishes matrices with integer 'I' and real 'R' elements, both actually stored as reals in double precision arithmetic. The second letter denotes randomly generated matrices ' $R$ ' or matrices determined by an explicit formula 'D'. For randomly generated matrices, a number specifying the interval for the random number generator follows, while the name of matrices determined by the explicit formula contains the formula number (F1, F2, F3). The last letter of the name denotes a way for obtaining ill-conditioned matrices: 'R' - matrices with nearly dependent rows, 'C' - matrices with nearly dependent columns, 'S' - nearly singular symmetric matrices, 'B' - both matrices in KKT system ill-conditioned. More specifically:

IR500 Randomly generated matrices with integer elements uniformly distributed in the interval $[-500,500]$.

IR500R Randomly generated matrices with integer elements uniformly distributed in the interval [-500,500] perturbed in addition to have two rows nearly dependent.

IR500C Randomly generated matrices with integer elements uniformly distributed in the interval $[-500,500]$ perturbed in addition to have two columns nearly dependent.

RR100 Randomly generated matrices with real elements uniformly distributed in the interval. $[-100,100]$

IDF1 Matrices with elements $a_{i j}=|i-j|, 1 \leq i \leq m, 1 \leq j \leq n$ (Micchelli-Fiedler matrix).

IDF2 Matrices with elements $a_{i j}=|i-j|^{2}, 1 \leq i \leq m, 1 \leq j \leq n$.

IDF3 Matrices with elements $a_{i j}=|i+j-(m+n) / 2|, 1, \leq i \leq m, 1 \leq j \leq n$.

IR50 Randomly generated matrices with integer elements uniformly distributed in the interval $[-50,50]$.

Matrices with linearly dependent rows were obtained in the following way. The input data contain four integers which specify two row indices $i_{1}, i_{2}$, one column index $i_{3}$ and one exponent $i_{4}$. Then the row $a_{i_{1}}$ is copied into $a_{i_{2}}$. Furthermore $a_{i_{1} i_{3}}$ is set to zero and $a_{i_{2} i_{3}}$ to $2^{-i_{4}}$. Similar procedures are used for columns and symmetric matrices.

Right hand sides were determined from the given solution vectors $x^{\star}$ by the formula $b=$ $A x^{\star}$. Solution vectors were usually generated randomly with integer or real elements uniformly distributed in the interval $[-10,10]$.

We have tested KKT systems with $m>>n / 2, m=n / 2$ and $m<<n / 2$. These systems were solved by using the modified Huang algorithm applied to the coupled system (7)-(8) and by two variants of the implicit LU algorithm with explicit column interchanges. The first variant is intended for solving the coupled system (7), (9) while the second one uses (7) together with the 
transformed system (11). For comparison, we have tested three additional methods implemented by using the LAPACK routines.

The first method, denoted by lu lapack, is in fact a direct solution of a complete KKT system by using the Bunch-Parlett decomposition. This is carried-out by the LAPACK routine DSPSV. Notice that we deal with an $n+m$ dimensional indefinite system in this case.

Another approach, a range-space method, is based on the Bunch-Parlett decomposition of the (possibly indefinite) matrix $B$. Using the LAPACK routine DSPSV, we obtain the solution of the matrix equation $B\left[\tilde{A}^{T}, \tilde{b}\right]=\left[A^{T}, b\right]$ together with the decomposition $B=L D L^{T}$, where $L$ is $n$-dimensional lower unit triangular and $D$ is $n$-dimensional block diagonal with the blocksize 1 or 2 . Then the symmetric matrix $C=A \tilde{A}^{T}=A B^{-1} A^{T}$ is built and the solution $y$ to the equation $C y=\tilde{b}-c$ is found, again by using the LAPACK routine DSPSV. Finaly, we solve the system $L D L^{T} x=b-A^{T} y$ by using the LAPACK routine DSPTRS.

The last approach, a null-space method, is based on the RQ decomposition

$$
A=[0, R] Q,
$$

where $R$ is a $m$-dimensional upper triangular matrix and $Q$ is an $n$-dimensional orthogonal matrix. This RQ decomposition is obtained by using the LAPACK routine DQERQF. Premultiplying equation (6) by $Q$, we get $Q B Q^{T} Q x+Q A^{T} y=Q b$ or

$$
\left[\begin{array}{ll}
\tilde{B}_{11} & \tilde{B}_{12} \\
\tilde{B}_{21} & \tilde{B}_{22}
\end{array}\right]\left[\begin{array}{l}
\tilde{x}_{1} \\
\tilde{x}_{2}
\end{array}\right]+\left[\begin{array}{c}
0 \\
R^{T}
\end{array}\right] y=\left[\begin{array}{c}
\tilde{b}_{1} \\
\tilde{b}_{2}
\end{array}\right] .
$$

where $\tilde{B}=Q B Q^{T}, \tilde{b}=Q b$ and $\tilde{x}=Q x$. Matrices $\tilde{B}$ and $\tilde{b}$ are obtained by using the LAPACK routine DORMRQ. Now $R \tilde{x}_{2}=c$ by (7), (12) and $\tilde{B}_{11} \tilde{x}_{1}=\tilde{b}_{1}-\tilde{B}_{12} \tilde{x}_{2}$ by (13). The last equation is solved by using the LAPACK routine DSYSV. Finally $R^{T} y=\tilde{b}_{2}-\tilde{B}_{21} \tilde{x}_{1}-\tilde{B}_{22} \tilde{x}_{2}$ and $x=Q^{T} \tilde{x}$ can be obtained by the LAPACK routine DORMRQ.

Notice that ABS algorithms were implemented in their basic form without partitioning into block or other special adjustements serving for speed increase as done in the LAPACK software.

For each selected problem, the type of matrix and the dimension is given. Furthermore, both the solution and the residual errors together with the detected rank and the computational time are given for each tested algorithm. Computational experiments were performed on a Digital Unix Workstation in the double precision arithmetic (machine epsilon equal to about $10^{-16}$ ).

In the Appendix we give detailed results. The following tables give synthetic results for the 24 tested problems, the number at the intersection of the $i$-th row with the $k$-th column indicating how many times the algorithm at the heading of the $i$-th row gave a lower error than the algorithm at the heading of the $k$-th row (in case there is a second number, this counts the number of cases when difference was less that one percent). 


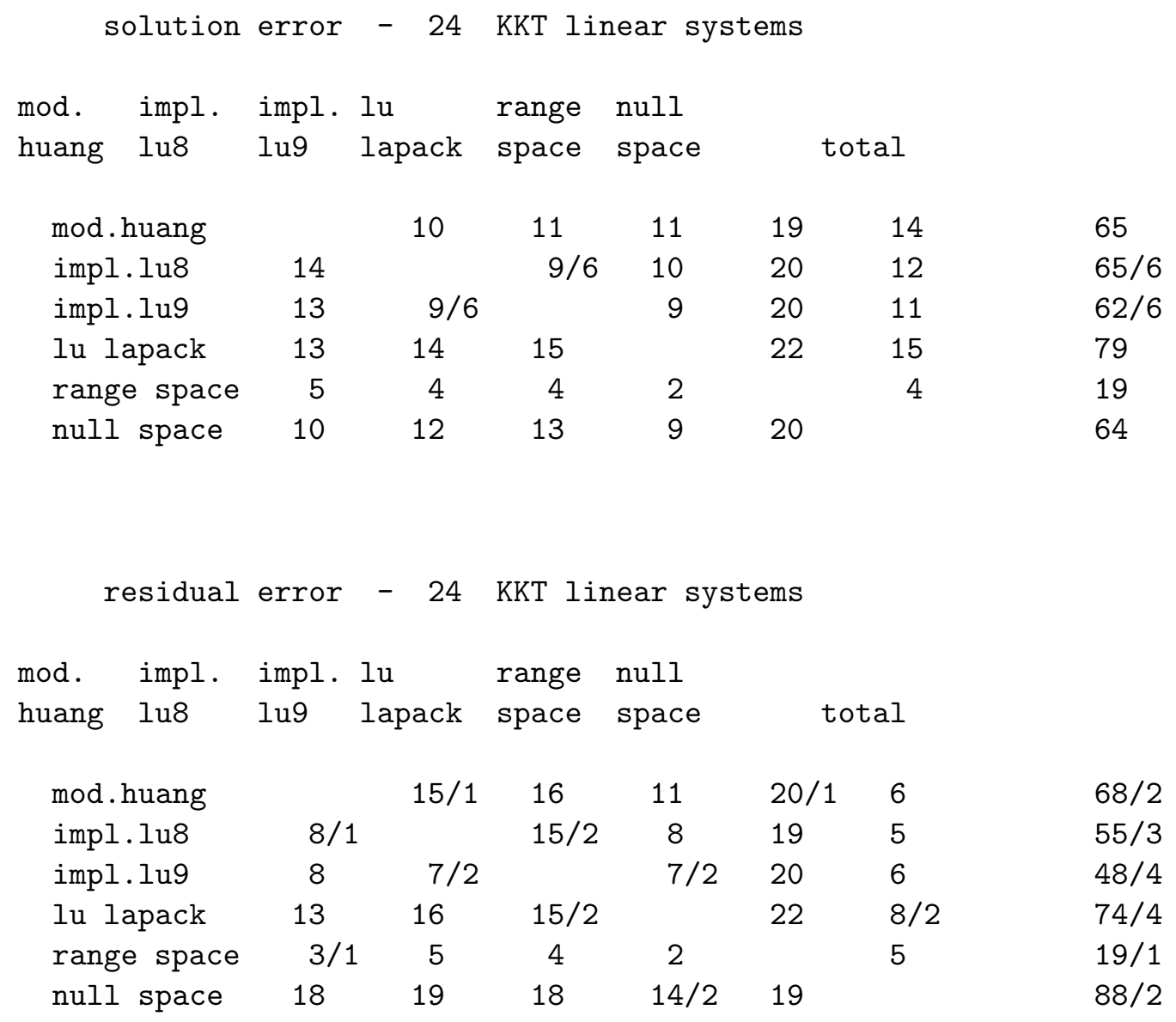

From the above tables and the results in the Appendix we can state the following conclusions:

(1) Implicit LU methods described in Subsection 2.3 are extremely suitable in term of total time cost for solving KKT systems with $m \sim n$. If $m<<n$, then a direct solution of a complete KKT system by using the Bunch-Parlett decomposition is faster.

(2) The method based on equation (9) is faster if $m \sim n$ while the method based on equation (11) is more efficient if $m<<n$.

(3) The modified Huang method is usually very slow. But in the extremely ill-conditioned cases it gives the best accuracy. Moreover, the modified Huang method is able to detect the numerical rank correctly, which can lead to the substantial decrease of the computational time as can be observed from experiments with the matrix IDF2.

(4) In term of accuracy the range space method is the least accurate; other methods have a comparable accuracy, with a marginal advantage for lu lapack (except for the very ill conditioned problems, where mod.huang gives the best results, up to four orders better). 


\section{References}

[1] J.Abaffy, C.G.Broyden, E.Spedicato: A class of direct methods for linear systems. Numerische Mathematik 45 (1984) pp. 361-376.

[2] J.Abaffy, E.Spedicato: ABS Projection Algorithms, Mathematical Techniques for solving Linear and Nonlinear Equations. Ellis Horwood, Chichester 1989.

[3] E.Bodon: Numerical results on the ABS algorithms for linear systems of equations. Rept. No. DMSIA 93/13, University of Bergamo, 1993.

[4] H.Y.Huang: A direct method for the general solution of a system of linear equations. JOTA 16 (1975) pp. 429-445.

[5] E.Spedicato: ABS algorithms for linear least squares: Theoretical results and computational performance. Rept. No. DMSIA 94/2, University of Bergamo, 1994.

[6] E.Spedicato, E.Bodon: Numerical behaviour of the implicit QR algorithm in the ABS class for linear least squares. Ricerca Operativa 22 (1992) pp.45-55.

[7] E.Spedicato, E.Bodon: Solving linear least squares by orthogonal factorization and pseudoinverse computation via the modified Huang algorithm in the ABS class. Computing 42 (1989) pp. 195-205.

[8] E.Spedicato, E.Bodon: Solution of linear least squares via the ABS algorithms. Mathematical Programming 58 (1993) pp. 111-136.

[9] E.Spedicato, Z.Chen, E.Bodon: ABS methods for KKT systems. In: Nonlinear Optimization and Applications (G.Di Pillo and F.Giannessi, eds), Plenum Press, New York 1996.

[10] E.Spedicato, H.Esmaeili, Z.Xia: A review of ABS algorithms for linear real and Diophantine equations and optimization. In: Proc. of the VI CMA Congresso de Matematica Aplicada (R.Montenegro, G.Montero, G.Winter, eds.), Las Palmas, Gran Canaria, September 1999.

[11] E.Spedicato, M.T.Vespucci: Variations on the Gram-Schmidt and the Huang algorithms for linear systems: a numerical study. Applications of Mathematics 2 (1223), 81-100.

[12] E. Bodon: ABS codes for linear KKT systems, Rept. No. DMASIA 25/00, University of Bergamo, 2000 


\section{Appendix: Test results for KKT linear systems}

\begin{tabular}{|c|c|c|c|c|c|c|c|}
\hline \multirow{2}{*}{$\begin{array}{c}\text { matrix } \\
\mathrm{n}\end{array}$} & \multicolumn{2}{|c|}{ dimension } & method & solution & \multirow[t]{2}{*}{ residual } & \multirow[t]{2}{*}{ rank } & \multirow[t]{2}{*}{ time } \\
\hline & $\mathrm{m}$ & & error & error & & & \\
\hline IR500 & 1000 & 900 & mod.huang & $0.50 \mathrm{D}-07$ & $0.36 \mathrm{D}-14$ & 1900 & 124.00 \\
\hline IR500 & 1000 & 900 & impl.lu8 & $0.70 \mathrm{D}-06$ & $0.16 \mathrm{D}-13$ & 1900 & 17.00 \\
\hline IR500 & 1000 & 900 & impl.lu9 & $0.71 \mathrm{D}-06$ & $0.18 D-13$ & 1900 & 19.00 \\
\hline IR500 & 1000 & 900 & lu lapack & $0.22 \mathrm{D}-05$ & $0.96 \mathrm{D}-14$ & 1900 & 56.00 \\
\hline IR500 & 1000 & 900 & range space & $0.42 \mathrm{D}-01$ & $0.52 \mathrm{D}-11$ & 1900 & 79.00 \\
\hline IR500 & 1000 & 900 & null space & $0.19 D-06$ & $0.20 \mathrm{D}-14$ & 1900 & 85.00 \\
\hline \multicolumn{3}{|c|}{ condition number: } & $0.22 \mathrm{D}+10$ & & & & \\
\hline IR500 & 1200 & 600 & mod.huang & $0.32 \mathrm{D}-07$ & $0.90 \mathrm{D}-14$ & 1800 & 188.00 \\
\hline IR500 & 1200 & 600 & impl.lu8 & $0.83 \mathrm{D}-07$ & $0.11 \mathrm{D}-13$ & 1800 & 44.00 \\
\hline IR500 & 1200 & 600 & impl.lu9 & $0.89 \mathrm{D}-07$ & $0.11 \mathrm{D}-13$ & 1800 & 33.00 \\
\hline IR500 & 1200 & 600 & lu lapack & $0.49 \mathrm{D}-07$ & $0.41 \mathrm{D}-14$ & 1800 & 47.00 \\
\hline IR500 & 1200 & 600 & range space & $0.23 \mathrm{D}-02$ & $0.55 D-11$ & 1800 & 64.00 \\
\hline IR500 & 1200 & 600 & null space & $0.55 \mathrm{D}-08$ & $0.26 \mathrm{D}-14$ & 1800 & 105.00 \\
\hline \multicolumn{3}{|c|}{ condition number: } & $0.15 \mathrm{D}+09$ & & & & \\
\hline IR500 & 1500 & 200 & mod.huang & $0.15 \mathrm{D}-07$ & $0.75 \mathrm{D}-14$ & 1700 & 303.00 \\
\hline IR500 & 1500 & 200 & impl.lu8 & $0.76 \mathrm{D}-08$ & $0.63 \mathrm{D}-14$ & 1700 & 78.00 \\
\hline IR500 & 1500 & 200 & impl.lu9 & $0.92 \mathrm{D}-08$ & $0.14 \mathrm{D}-13$ & 1700 & 59.00 \\
\hline IR500 & 1500 & 200 & lu lapack & $0.31 D-08$ & $0.91 \mathrm{D}-14$ & 1700 & 40.00 \\
\hline IR500 & 1500 & 200 & range space & $0.24 \mathrm{D}-05$ & $0.16 \mathrm{D}-12$ & 1700 & 41.00 \\
\hline IR500 & 1500 & 200 & null space & $0.19 D-08$ & $0.25 \mathrm{D}-14$ & 1700 & 78.00 \\
\hline \multicolumn{3}{|c|}{ condition number: } & $0.10 \mathrm{D}+08$ & & & & \\
\hline IR500R & 1000 & 900 & mod.huang & $0.30 D+01$ & $0.49 D-14$ & 1900 & 124.00 \\
\hline IR500R & 1000 & 900 & impl.lu8 & $0.80 \mathrm{D}-01$ & $0.14 \mathrm{D}-13$ & 1900 & 17.00 \\
\hline IR500R & 1000 & 900 & impl.lu9 & $0.80 \mathrm{D}-01$ & $0.10 \mathrm{D}-13$ & 1900 & 19.00 \\
\hline IR500R & 1000 & 900 & lu lapack & $0.22 \mathrm{D}+01$ & $0.10 \mathrm{D}-13$ & 1900 & 55.00 \\
\hline IR500R & 1000 & 900 & range space & $0.74 \mathrm{D}+00$ & $0.75 D-11$ & 1900 & 79.00 \\
\hline IR500R & 1000 & 900 & null space & $0.64 \mathrm{D}+01$ & $0.28 \mathrm{D}-14$ & 1900 & 85.00 \\
\hline \multicolumn{3}{|c|}{ condition number: } & $0.18 \mathrm{D}+17$ & & & & \\
\hline IR500R & 1200 & 600 & mod.huang & $0.15 \mathrm{D}+00$ & $0.81 \mathrm{D}-14$ & 1800 & 187.00 \\
\hline IR500R & 1200 & 600 & impl.lu8 & $0.35 \mathrm{D}-02$ & $0.12 \mathrm{D}-13$ & 1800 & 44.00 \\
\hline IR500R & 1200 & 600 & impl.lu9 & $0.35 \mathrm{D}-02$ & $0.14 \mathrm{D}-13$ & 1800 & 34.00 \\
\hline IR500R & 1200 & 600 & lu lapack & $0.57 \mathrm{D}+00$ & $0.64 \mathrm{D}-14$ & 1800 & 47.00 \\
\hline IR500R & 1200 & 600 & range space & $0.16 \mathrm{D}+01$ & $0.91 \mathrm{D}-11$ & 1800 & 63.00 \\
\hline IR500R & 1200 & 600 & null space & $0.46 \mathrm{D}+00$ & $0.20 \mathrm{D}-14$ & 1800 & 105.00 \\
\hline \multicolumn{3}{|c|}{ condition number: } & $0.15 \mathrm{D}+16$ & & & & \\
\hline IR500R & 1500 & 200 & mod.huang & $0.50 \mathrm{D}+00$ & $0.98 D-14$ & 1700 & 302.00 \\
\hline IR500R & 1500 & 200 & impl.lu8 & $0.81 \mathrm{D}-01$ & $0.61 \mathrm{D}-14$ & 1700 & 78.00 \\
\hline IR500R & 1500 & 200 & impl.lu9 & $0.81 \mathrm{D}-01$ & $0.83 \mathrm{D}-14$ & 1700 & 59.00 \\
\hline IR500R & 1500 & 200 & lu lapack & $0.13 D+01$ & $0.16 \mathrm{D}-13$ & 1700 & 39.00 \\
\hline IR500R & 1500 & 200 & range space & $0.27 \mathrm{D}+01$ & $0.36 \mathrm{D}-12$ & 1700 & 41.00 \\
\hline IR500R & 1500 & 200 & null space & $0.19 D+00$ & $0.31 \mathrm{D}-14$ & 1700 & 78.00 \\
\hline \multicolumn{3}{|c|}{ condition number: } & $0.17 \mathrm{D}+16$ & & & & \\
\hline
\end{tabular}


Test results for KKT linear systems - continued

\begin{tabular}{|c|c|c|c|c|c|c|c|}
\hline matrix & dimen & sion & method & solution & residual & rank & time \\
\hline $\mathrm{n}$ & $\mathrm{m}$ & & error & error & & & \\
\hline IR500S & 1000 & 900 & mod.huang & $0.31 D-06$ & $0.36 \mathrm{D}-14$ & 1900 & 125.00 \\
\hline IR500S & 1000 & 900 & impl.lu8 & $0.41 \mathrm{D}-04$ & $0.14 D-13$ & 1900 & 17.00 \\
\hline IR500S & 1000 & 900 & impl.lu9 & $0.41 \mathrm{D}-04$ & $0.30 D-13$ & 1900 & 21.00 \\
\hline IR500S & 1000 & 900 & lu lapack & $0.75 \mathrm{D}-05$ & $0.19 D-14$ & 1900 & 56.00 \\
\hline IR500S & 1000 & 900 & range space & $0.34 D+02$ & $0.37 D+01$ & 1900 & 80.00 \\
\hline IR500S & 1000 & 900 & null space & $0.11 \mathrm{D}-04$ & $0.19 D-14$ & 1900 & 89.00 \\
\hline conditio & on numb & er: & $0.14 D+12$ & & & & \\
\hline IR500S & 1200 & 600 & mod.huang & $0.57 \mathrm{D}-08$ & $0.37 D-13$ & 1800 & 189.00 \\
\hline IR500S & 1200 & 600 & impl.lu8 & $0.12 \mathrm{D}-06$ & $0.43 D-12$ & 1800 & 42.00 \\
\hline IR500S & 1200 & 600 & impl.lu9 & $0.15 \mathrm{D}-06$ & $0.16 \mathrm{D}-11$ & 1800 & 34.00 \\
\hline IR500S & 1200 & 600 & lu lapack & $0.18 \mathrm{D}-08$ & $0.19 D-13$ & 1800 & 49.00 \\
\hline IR500S & 1200 & 600 & range space & $0.18 D+02$ & $0.22 \mathrm{D}+01$ & 1800 & 65.00 \\
\hline IR500S & 1200 & 600 & null space & $0.23 \mathrm{D}-07$ & $0.19 D-13$ & 1800 & 110.00 \\
\hline conditio & n numb & er: & $0.23 \mathrm{D}+09$ & & & & \\
\hline IR500S & 1500 & 200 & mod.huang & $0.23 D-08$ & $0.76 \mathrm{D}-14$ & 1700 & 304.00 \\
\hline IR500S & 1500 & 200 & impl.lu8 & $0.13 \mathrm{D}-07$ & $0.91 \mathrm{D}-14$ & 1700 & 78.00 \\
\hline IR500S & 1500 & 200 & impl.lu9 & $0.12 \mathrm{D}-07$ & $0.14 D-14$ & 1700 & 60.00 \\
\hline IR500S & 1500 & 200 & lu lapack & $0.15 \mathrm{D}-08$ & $0.11 \mathrm{D}-14$ & 1700 & 41.00 \\
\hline IR500S & 1500 & 200 & range space & $0.14 D+03$ & $0.53 D+02$ & 1700 & 43.00 \\
\hline IR500S & 1500 & 200 & null space & $0.95 \mathrm{D}-08$ & $0.19 D-14$ & 1700 & 77.00 \\
\hline conditio & n numb & er: & $0.30 \mathrm{D}+08$ & & & & \\
\hline IR500B & 1000 & 900 & mod.huang & $0.12 \mathrm{D}-01$ & $0.83 D-14$ & 1900 & 125.00 \\
\hline IR500B & 1000 & 900 & impl.1u8 & $0.14 \mathrm{D}-02$ & $0.13 D-13$ & 1900 & 17.00 \\
\hline IR500B & 1000 & 900 & impl.lu9 & $0.14 \mathrm{D}-02$ & $0.14 \mathrm{D}-13$ & 1900 & 20.00 \\
\hline IR500B & 1000 & 900 & lu lapack & $0.17 \mathrm{D}-02$ & $0.45 D-14$ & 1900 & 58.00 \\
\hline IR500B & 1000 & 900 & range space & $0.26 \mathrm{D}+03$ & $0.13 \mathrm{D}-02$ & 1900 & 78.00 \\
\hline IR500B & 1000 & 900 & null space & $0.17 \mathrm{D}-01$ & $0.23 D-14$ & 1900 & 88.00 \\
\hline conditio & n numb & er: & $0.76 \mathrm{D}+14$ & & & & \\
\hline IR500B & 1200 & 600 & mod.huang & $0.69 \mathrm{D}-03$ & $0.60 \mathrm{D}-14$ & 1800 & 189.00 \\
\hline IR500B & 1200 & 600 & impl.lu8 & $0.15 \mathrm{D}-03$ & $0.10 D-13$ & 1800 & 43.00 \\
\hline IR500B & 1200 & 600 & impl.lu9 & $0.15 \mathrm{D}-03$ & $0.11 \mathrm{D}-13$ & 1800 & 33.00 \\
\hline IR500B & 1200 & 600 & lu lapack & $0.38 \mathrm{D}-02$ & $0.20 D-13$ & 1800 & 47.00 \\
\hline IR500B & 1200 & 600 & range space & $0.66 \mathrm{D}+03$ & $0.22 \mathrm{D}-03$ & 1800 & 62.00 \\
\hline IR500B & 1200 & 600 & null space & $0.11 \mathrm{D}-01$ & $0.21 \mathrm{D}-14$ & 1800 & 108.00 \\
\hline conditio & n numb & er: & $0.24 \mathrm{D}+14$ & & & & \\
\hline IR500B & 1500 & 200 & mod.huang & $0.89 D-03$ & $0.11 \mathrm{D}-13$ & 1700 & 309.00 \\
\hline IR500B & 1500 & 200 & impl.lu8 & $0.23 \mathrm{D}-05$ & $0.11 \mathrm{D}-13$ & 1700 & 77.00 \\
\hline IR500B & 1500 & 200 & impl.lu9 & $0.16 \mathrm{D}-05$ & $0.40 \mathrm{D}-13$ & 1700 & 57.00 \\
\hline IR500B & 1500 & 200 & lu lapack & $0.15 \mathrm{D}-03$ & $0.42 \mathrm{D}-14$ & 1700 & 39.00 \\
\hline IR500B & 1500 & 200 & range space & $0.22 \mathrm{D}+04$ & $0.12 \mathrm{D}+00$ & 1700 & 39.00 \\
\hline IR500B & 1500 & 200 & null space & $0.18 D-02$ & $0.34 D-14$ & 1700 & 77.00 \\
\hline conditic & numk & & $0.19 D+13$ & & & & \\
\hline
\end{tabular}


Test results for KKT linear systems - continued

\begin{tabular}{|c|c|c|c|c|c|c|c|}
\hline \multirow{2}{*}{$\underset{n}{\operatorname{matrix}}$} & \multicolumn{2}{|c|}{ dimension } & method & solution & \multirow[t]{2}{*}{ residual } & \multirow[t]{2}{*}{ rank } & \multirow[t]{2}{*}{ time } \\
\hline & $\mathrm{m}$ & & error & error & & & \\
\hline RR100 & 1000 & 900 & mod.huang & $0.57 \mathrm{D}-10$ & $0.18 D-12$ & 1900 & 136.00 \\
\hline RR100 & 1000 & 900 & impl.lu8 & $0.64 \mathrm{D}-12$ & $0.13 D-13$ & 1900 & 18.00 \\
\hline RR100 & 1000 & 900 & impl.lu9 & $0.50 D-12$ & $0.23 D-13$ & 1900 & 21.00 \\
\hline RR100 & 1000 & 900 & lu lapack & $0.23 D-11$ & $0.11 \mathrm{D}-12$ & 1900 & 60.00 \\
\hline RR100 & 1000 & 900 & range space & $0.18 D-08$ & $0.10 D-10$ & 1900 & 87.00 \\
\hline RR100 & 1000 & 900 & null space & $0.23 D-12$ & $0.26 \mathrm{D}-14$ & 1900 & 91.00 \\
\hline \multicolumn{3}{|c|}{ condition number: } & $0.13 \mathrm{D}+04$ & & & & \\
\hline RR100 & 1200 & 600 & mod.huang & $0.92 D-10$ & $0.83 D-12$ & 1800 & 187.00 \\
\hline RR100 & 1200 & 600 & impl.lu8 & $0.13 D-11$ & $0.26 \mathrm{D}-13$ & 1800 & 44.00 \\
\hline RR100 & 1200 & 600 & impl.lu9 & $0.16 \mathrm{D}-11$ & $0.70 D-13$ & 1800 & 34.00 \\
\hline RR100 & 1200 & 600 & lu lapack & $0.15 D-11$ & $0.11 \mathrm{D}-12$ & 1800 & 47.00 \\
\hline RR100 & 1200 & 600 & range space & $0.25 D-09$ & $0.19 D-11$ & 1800 & 64.00 \\
\hline RR100 & 1200 & 600 & null space & $0.88 \mathrm{D}-13$ & $0.24 D-14$ & 1800 & 105.00 \\
\hline \multicolumn{3}{|c|}{ condition number: } & $0.50 \mathrm{D}+03$ & & & & \\
\hline RR100 & 1500 & 200 & mod.huang & $0.23 D-08$ & $0.36 \mathrm{D}-10$ & 1700 & 301.00 \\
\hline RR100 & 1500 & 200 & impl.lu8 & $0.10 D-11$ & $0.42 \mathrm{D}-13$ & 1700 & 78.00 \\
\hline RR100 & 1500 & 200 & impl.lu9 & $0.33 D-11$ & $0.10 D-12$ & 1700 & 59.00 \\
\hline RR100 & 1500 & 200 & lu lapack & $0.62 \mathrm{D}-12$ & $0.11 \mathrm{D}-12$ & 1700 & 39.00 \\
\hline RR100 & 1500 & 200 & range space & $0.28 D-10$ & $0.87 D-12$ & 1700 & 41.00 \\
\hline RR100 & 1500 & 200 & null space & $0.96 \mathrm{D}-13$ & $0.23 D-14$ & 1700 & 78.00 \\
\hline \multicolumn{3}{|c|}{ condition number: } & $0.15 \mathrm{D}+03$ & & & & \\
\hline IDF1 & 1000 & 900 & mod.huang & $0.85 D-10$ & $0.18 D-14$ & 1900 & 133.00 \\
\hline IDF1 & 1000 & 900 & impl.lu8 & $0.38 D-10$ & $0.13 \mathrm{D}-14$ & 1900 & 17.00 \\
\hline IDF 1 & 1000 & 900 & impl.lu9 & $0.36 \mathrm{D}-10$ & $0.14 D-14$ & 1900 & 20.00 \\
\hline IDF 1 & 1000 & 900 & lu lapack & $0.48 D-10$ & $0.35 D-14$ & 1900 & 59.00 \\
\hline IDF 1 & 1000 & 900 & range space & $0.79 D-10$ & $0.18 D-14$ & 1900 & 85.00 \\
\hline IDF 1 & 1000 & 900 & null space & $0.66 \mathrm{D}-10$ & $0.21 \mathrm{D}-14$ & 1900 & 92.00 \\
\hline \multicolumn{3}{|c|}{ condition number: } & $0.80 \mathrm{D}+04$ & & & & \\
\hline IDF 1 & 1200 & 600 & mod.huang & $0.16 \mathrm{D}-08$ & $0.27 D-13$ & 1800 & 182.00 \\
\hline IDF1 & 1200 & 600 & impl.lu8 & $0.55 \mathrm{D}-10$ & $0.15 D-14$ & 1800 & 43.00 \\
\hline IDF1 & 1200 & 600 & impl.lu9 & $0.49 \mathrm{D}-10$ & $0.15 \mathrm{D}-14$ & 1800 & 33.00 \\
\hline IDF 1 & 1200 & 600 & lu lapack & $0.28 D-10$ & $0.19 \mathrm{D}-14$ & 1800 & 47.00 \\
\hline IDF 1 & 1200 & 600 & range space & $0.87 D-10$ & $0.16 \mathrm{D}-14$ & 1800 & 63.00 \\
\hline IDF1 & 1200 & 600 & null space & $0.64 \mathrm{D}-10$ & $0.25 \mathrm{D}-14$ & 1800 & 105.00 \\
\hline \multicolumn{3}{|c|}{ condition number: } & $0.96 \mathrm{D}+04$ & & & & \\
\hline IDF 1 & 1500 & 200 & mod.huang & $0.33 D-07$ & $0.42 D-12$ & 1700 & 303.00 \\
\hline IDF1 & 1500 & 200 & impl.lu8 & $0.52 \mathrm{D}-10$ & $0.19 D-14$ & 1700 & 77.00 \\
\hline IDF1 & 1500 & 200 & impl.lu9 & $0.63 D-10$ & $0.15 D-14$ & 1700 & 58.00 \\
\hline IDF1 & 1500 & 200 & lu lapack & $0.28 \mathrm{D}-10$ & $0.15 D-14$ & 1700 & 39.00 \\
\hline IDF1 & 1500 & 200 & range space & $0.11 \mathrm{D}-09$ & $0.18 D-14$ & 1700 & 41.00 \\
\hline IDF1 & 1500 & 200 & null space & $0.15 \mathrm{D}-09$ & $0.33 D-14$ & 1700 & 78.00 \\
\hline \multicolumn{3}{|c|}{ condition number: } & $0.12 \mathrm{D}+05$ & & & & \\
\hline
\end{tabular}


Test results for KKT linear systems - continued

\begin{tabular}{|c|c|c|c|c|c|c|c|}
\hline \multirow{2}{*}{$\begin{array}{l}\text { matrix } \\
\mathrm{n}\end{array}$} & \multirow{2}{*}{\multicolumn{2}{|c|}{ m dimension }} & \multirow{2}{*}{$\begin{array}{l}\text { method } \\
\text { error }\end{array}$} & solution & \multirow{2}{*}{ residual } & \multirow[t]{2}{*}{ rank } & \multirow[t]{2}{*}{ time } \\
\hline & & & & error & & & \\
\hline IDF2 & 1000 & 900 & mod.huang & $0.55 \mathrm{D}+01$ & $0.23 D-14$ & 16 & 24.00 \\
\hline IDF2 & 1000 & 900 & impl.lu8 & $0.44 D+13$ & $0.21 \mathrm{D}-03$ & 1900 & 18.00 \\
\hline IDF2 & 1000 & 900 & impl.lu9 & $0.12 D+15$ & $0.80 \mathrm{D}-02$ & 1900 & 21.00 \\
\hline IDF2 & 1000 & 900 & lu lapack & $0.25 \mathrm{D}+03$ & $0.31 \mathrm{D}-13$ & 1900 & 62.00 \\
\hline IDF2 & 1000 & 900 & range space & $0.16 \mathrm{D}+05$ & $0.14 \mathrm{D}-11$ & 1900 & 87.00 \\
\hline IDF2 & 1000 & 900 & null space & $0.89 D+03$ & $0.15 \mathrm{D}-12$ & 1900 & 93.00 \\
\hline \multicolumn{3}{|c|}{ condition number: } & $0.26 \mathrm{D}+21$ & & & & \\
\hline IDF2 & 1200 & 600 & mod.huang & $0.62 \mathrm{D}+01$ & $0.20 \mathrm{D}-14$ & 17 & 36.00 \\
\hline IDF2 & 1200 & 600 & impl.lu8 & $0.22 \mathrm{D}+07$ & $0.10 \mathrm{D}-08$ & 1800 & 44.00 \\
\hline IDF2 & 1200 & 600 & impl.lu9 & $0.21 \mathrm{D}+06$ & $0.56 \mathrm{D}-09$ & 1800 & 33.00 \\
\hline IDF2 & 1200 & 600 & lu lapack & $0.10 D+03$ & $0.79 D-14$ & 1800 & 47.00 \\
\hline IDF2 & 1200 & 600 & range space & $0.11 \mathrm{D}+05$ & $0.15 \mathrm{D}-11$ & 1800 & 63.00 \\
\hline IDF2 & 1200 & 600 & null space & $0.38 D+04$ & $0.13 \mathrm{D}-12$ & 1800 & 105.00 \\
\hline \multicolumn{3}{|c|}{ condition number: } & $0.70 \mathrm{D}+20$ & & & & \\
\hline IDF2 & 1500 & 200 & mod.huang & $0.38 D+01$ & $0.22 \mathrm{D}-14$ & 17 & 68.00 \\
\hline IDF2 & 1500 & 200 & impl.lu8 & $0.31 D+04$ & $0.76 \mathrm{D}-11$ & 1700 & 79.00 \\
\hline IDF2 & 1500 & 200 & impl.lu9 & $0.58 D+03$ & $0.57 D-12$ & 1700 & 59.00 \\
\hline IDF2 & 1500 & 200 & lu lapack & $0.28 D+03$ & $0.19 \mathrm{D}-13$ & 1700 & 39.00 \\
\hline IDF2 & 1500 & 200 & range space & $0.99 \mathrm{D}+05$ & $0.16 \mathrm{D}-10$ & 1700 & 41.00 \\
\hline IDF2 & 1500 & 200 & null space & $0.25 D+03$ & $0.36 \mathrm{D}-13$ & 1700 & 78.00 \\
\hline \multicolumn{3}{|c|}{ condition number: } & $0.28 \mathrm{D}+20$ & & & & \\
\hline IR50 & 1000 & 900 & mod.huang & $0.76 \mathrm{D}+00$ & $0.48 D-14$ & 1702 & 143.00 \\
\hline IR50 & 1000 & 900 & impl.lu8 & $0.29 D+16$ & $0.28 \mathrm{D}+00$ & 1900 & 18.00 \\
\hline IR50 & 1000 & 900 & impl.lu9 & $0.13 D+16$ & $0.15 \mathrm{D}+00$ & 1900 & 21.00 \\
\hline IR50 & 1000 & 900 & lu lapack & $0.10 D+04$ & $0.59 D-13$ & 1900 & 60.00 \\
\hline IR50 & 1000 & 900 & range space & $0.24 D+03$ & $0.17 D-10$ & 1900 & 84.00 \\
\hline IR50 & 1000 & 900 & null space & $0.18 D+18$ & $0.11 \mathrm{D}+02$ & 1900 & 91.00 \\
\hline \multicolumn{3}{|c|}{ condition number: } & $0.27 \mathrm{D}+19$ & & & & \\
\hline IR50 & 1200 & 600 & mod.huang & $0.30 D+00$ & $0.84 \mathrm{D}-14$ & 1751 & 186.00 \\
\hline IR50 & 1200 & 600 & impl.lu8 & $0.44 D+14$ & $0.53 \mathrm{D}-02$ & 1800 & 44.00 \\
\hline IR50 & 1200 & 600 & impl.lu9 & $0.23 D+14$ & $0.33 D-02$ & 1800 & 34.00 \\
\hline IR50 & 1200 & 600 & lu lapack & $0.63 \mathrm{D}+02$ & $0.15 \mathrm{D}-13$ & 1800 & 47.00 \\
\hline IR50 & 1200 & 600 & range space & $0.10 \mathrm{D}+03$ & $0.28 \mathrm{D}-09$ & 1800 & 63.00 \\
\hline IR50 & 1200 & 600 & null space & $0.82 D+15$ & $0.66 \mathrm{D}-01$ & 1800 & 105.00 \\
\hline \multicolumn{3}{|c|}{ condition number: } & $0.14 D+18$ & & & & \\
\hline IR50 & 1500 & 200 & mod.huang & $0.33 \mathrm{D}-07$ & $0.68 \mathrm{D}-14$ & 1700 & 302.00 \\
\hline IR50 & 1500 & 200 & impl.lu8 & $0.73 D-08$ & $0.76 \mathrm{D}-14$ & 1700 & 78.00 \\
\hline IR50 & 1500 & 200 & impl.lu9 & $0.63 \mathrm{D}-07$ & $0.99 \mathrm{D}-14$ & 1700 & 59.00 \\
\hline IR50 & 1500 & 200 & lu lapack & $0.58 D-09$ & $0.63 \mathrm{D}-14$ & 1700 & 39.00 \\
\hline IR50 & 1500 & 200 & range space & $0.20 \mathrm{D}-05$ & $0.36 \mathrm{D}-13$ & 1700 & 41.00 \\
\hline IR50 & 1500 & 200 & null space & $0.11 \mathrm{D}-07$ & $0.36 \mathrm{D}-14$ & 1700 & 78.00 \\
\hline \multicolumn{3}{|c|}{ condition number: } & $0.12 \mathrm{D}+08$ & & & & \\
\hline
\end{tabular}

